\title{
ABO Blood Groups and Its Association with Oral Cancer, Oral Potentially Malignant Disorders and Oral Submucous Fibrosis- A Systematic Review and Meta-Analysis
}

\author{
Abhinav Singh ${ }^{1 *}$, Bharathi M Purohit ${ }^{2}$
}

\begin{abstract}
Objective: The study aimed to evaluate the association between $\mathrm{ABO}$ blood groups and oral cancer, other potentially malignant disorders (OPMD), and oral submucous fibrosis (OSMF). Materials and methods: A search was conducted in Medline, Cochrane databases, Google Scholar, Scopus, Web of Science and Directory of Open Access Journals (DOAJ) for studies evaluating ABO blood groups as risk factors for oral cancer and OPMD among cases and controls. The PRISMA guidelines were followed for the meta-analysis. Participants included patients with oral cancer, and OPMD diagnosed using histopathologic investigations. Sub-group analysis was conducted to evaluate the association between blood groups and OSMF. Quality was evaluated using the Risk of Bias Assessment tool. Fixed effects model was used to assess the odds ratio for the association. Results: There were 1,352, 414, and 299 cases of oral cancer, OPMD, and OSMF and 11,699, 7,382 and 7,307 controls for analysis respectively. Blood group A was significantly associated with both oral cancer (Odds ratio: 1.27 [95\% CI, 1.10, 1.47], $\mathrm{P}=0.001$ ) and OPMD (Odds ratio: 1.33 [95\% $\mathrm{CI}, 1.01,1.47], \mathrm{P}=0.04)$. No association was noted between blood group $\mathrm{B}$ and $\mathrm{AB}$ with oral cancer, OPMD, and OSMF. Blood group $\mathrm{O}$ was significantly associated with lower chances of oral cancer (Odds ratio: 0.81 [95\% CI, 0.71, 0.93], $\mathrm{P}=0.002$ ). Conclusion: Meta-analysis suggests blood group A has a greater risk for developing oral cancer and OPMD. Blood group O was associated with lower chances of oral cancer. No association was noted between ABO blood group system with OSMF.
\end{abstract}

Keywords: ABO blood group system- oral cancer- potentially malignant disorders- oral submucous fibrosis

Asian Pac J Cancer Prev, 22 (6), 1703-1712

\section{Introduction}

Oral cancer is one of the widely prevalent malignant diseaseand a major public health problem. There are an estimated 657,000 new cases of oral cancers each year, and more than 330,000 deaths (WHO, 2020). Oral and pharyngeal cancer, grouped together is the $6^{\text {th }}$ most common cancer in the world and the most common head and neck cancer in India (Warnakulasuriya, 2009; ICMR and NCDIR, 2020). Majority of the global incidence of oral cancer occurs in developing countries; half of those cases are in South Asia. India reports for more than one-fourth of all oral cancer deathswith an age-standardized incidence rate of 12.6 per lakh population and is estimated to record a $12 \%$ rise in cancer by 2025 (Sankaranarayanan et al., 2015; ICMR and NCDIR, 2020).

World Health Organization in 2005 suggested a term oral potentially malignant disorders (OPMD) and defined it as the risk of malignancy being present in a lesion or condition either at the time of initial diagnosis or at a future date (Wanakulasuriya et al., 2007). Oral hypoplasia, dysplasia, leukoplakia, erythroplakia and oral submucous fibrosis were included under broad term OPMD. OPMD showed a high risk of malignant transformation and the observed rates ranged from $0.6 \%$ to $36 \%$ (Shah et al., 2008; Dadhich et al., 2015).

The increasing number of oral malignancy and potentially malignant disorders related morbidity and mortality has been related to its multi-factorial aetiology; besides lifestyle and environmental factors, genetics and heredity have important role in causing cancer. Tobacco use and alcohol consumption account for more than 90 percent of oral cancers. Approximately 5 to 10 percent of cases are entirely hereditary (Warnakulasuriya, 2009). The ABO blood group is one of the genetic factors that has been hypothesized as the risk factors for different type of cancers.

The possibility of association between ABO blood groups and malignancy was first explored by Anderson and Haas (Anderson and Haas, 1984). Association of blood 
Abhinav Singh and Bharathi M Purohit

Group A in various cancers, including gastric cancers, breast cancers, pancreatic cancers, skin cancers, ovary and oesophageal cancers has been reported in various cohort studies and systematic reviews (Vasan et al., 2016; Mao et al., 2019; Miao et al., 2014; Celic et al., 2019; Huang et al., 2015). The association between ABO blood type with oral cancer and other potentially malignant disorders has also been studied but has yet not been established as risk factors due to contradictory findings (Gupta et al., 2020; Mortazavi et al., 2014; Jaleel and Nagarajappa, 2012; Jalili et al., 2018; Jamil et al., 2006; Kumari et al., 2017; Oroei, 2019; Poornima et al., 2018; Shishodia et al., 2018; Singh et al., 2014; Bhateja et al., 2014).

Blood group antigens in humans are present on the surface of red blood cells and epithelial cells of tissues such as mucosa and body fluids. The mechanism hypothesized behind the association between blood group $\mathrm{A}$ and carcinomas is that the carcinoma cells produce an antigen immunologically related to blood group A which predominantly in $\mathrm{O}$ group individuals may have a shielding effect by checking the growth and spread of the tumor. People with blood groups A and AB lack antibodies to A and are more prone to develop these carcinomas (Beckman and Angqvist, 1987; Henderson et al., 1993). Loss of expression of A or B antigens in more than $80 \%$ of cases of OSCC and OPMD have also been reported by immune-histochemical evaluations (Dabelsteen and Gao, 2015).

Numerous studies have been conducted to assess $\mathrm{ABO}$ blood group and its association with oral cancer, other potentially malignant disorders, and oral submucous fibrosis (Gupta et al., 2020; Mortazavi et al., 2014; Jaleel and Nagarajappa, 2012; Jalili et al., 2018; Jamil et al., 2006; Kumari et al., 2017; Oroei, 2019; Poornima et al., 2018; Shishodia et al., 2018; Singh et al., 2014; Bhateja et al., 2014). However, evidence from these studies remains inconclusive. This paper is a meta-analysis which aims to evaluate whether any ABO blood group types have a higher predisposition for oral cancer, OPMD, and OSMF. The study aims to determine the association between oral cancer, OPMD, and OSMF. The Population Exposure Comparison Outcome (PECO) question was population: participants were patients with oral cancer, OPMD, and OSMF diagnosed using histopathologic investigations; exposure to the risk factor: ABO blood grouping; comparison: healthy population with no oral cancer, OPMD, and OSMF; and outcome: prevalence of oral cancer, OPMD, and OSMF.

\section{Materials and Methods}

\section{Inclusion criteria}

Inclusion and exclusion criteria were determined before the literature search. All observational studies including case-control, prospective and retrospective studies evaluating the association between ABO blood group among patients with oral cancer, oral potentially malignant disorders (OPMD), and oral submucous fibrosis (OSMF) as cases with healthy participants as controls were included. Participants were patients confirmed using histopathologic investigations. Exposure to the risk factor was ABO blood grouping among cases and controls. Outcome was the prevalence of oral cancer, OPMD, and OSMF. In vitro experiments or experiments involving laboratory animals were excluded.

\section{Search strategy}

A search was conducted in Medline, Cochrane databases, Google Scholar, Scopus, Web of Science and Directory of Open Access Journals (DOAJ) for studies through May 2021. PRISMA guidelines were followed for the meta-analysis. Literature search keywords were: ABO blood group system AND oral cancer OR precancer OR oral sub mucous fibrosis. Boolean operators used with keywords are attached as an appendix. Studies were selected independently by two investigators (AS and BP). The title and abstracts were pre-screened to decide if the studies would be retrieved in full and to exclude ineligible studies. Retrieved articles were read before they were included in the review. Differences between the two investigators were resolved by discussion. A third person with subject expertise had been pre-approved by the two investigators in case of lack of consensus. References in the selected papers were manually reviewed and retrieved if they were relevant. The articles were searched using English keywords. No restrictions were placed on the publication language. Grey literature was searched through unpublished articles and manual searching of non-indexed journals at the institutional library at All India Institute of Medical Sciences (AIIMS), New Delhi (WHO Collaborating Centre for Oral Health Promotion) and AIIMS, Bhopal and through abstracts, conference presentations, online clinical registries including results of completed but unpublished trials for inclusion in the review.

\section{Data extraction and quality assessment}

Dichotomous outcome data for the absence or presence of oral cancer, oral potentially malignant disorders (OPMD), and oral submucous fibrosis (OSMF) was extracted from the included studies in a pilot tested worksheet. The following variables were extracted from each study: first author's name, publication year, country of origin, study design, numbers of cases and controls in different $\mathrm{ABO}$ blood types. Outcome data were extracted independently by the two investigators (AS and BP) using guidelines published by the Cochrane Collaboration (The Cochrane Collaboration, 2011). Differences between the two investigators were resolved by discussion. Characteristics of the studies that were included in the meta-analysis are presented in Table 1. Quality was evaluated by both the investigators using the Risk of Bias Assessment tool for Non-randomized Studies (Kim et al., 2013). Six domains (participant selection, confounding variables, exposure measurement, attrition for prospective studies, incomplete outcome data, and selective outcome reporting) were evaluated according to a low, moderate, and high risk of bias (Table 3). Attrition was to be reported only for prospective studies.

Certainty of evidence was evaluated using GRADE assessment (a systematic approach to rating the certainty of evidence in systematic reviews and other evidence 
syntheses) (The GRADE Handbook, 2013). Explicit consideration was given to the type of included studies, risk of bias, consistency, directness of evidence, precision of results, risk of publication bias, magnitude of the effect, and influence of residual plausible confounding factors. The GRADE approach assesses the quality of a body of evidence as high, moderate, low, or very low.

\section{Analysis}

A systematic review was conducted using Cochrane Program Review Manager Version 5. A fixed effects model was used to assess the association between ABO blood groups and oral cancer and OPMD. Blood groups are fixed in an individual and are the independent variables, the dependent variable changes in response to the level of independent variables. Fixed effect model was used as the effect size was assumed to be fixed for different blood groups, irrespective of study location. Sub-group analysis was conducted to evaluate the association between blood groups and OSMF. The odds ratio with $95 \%$ confidence interval was calculated to evaluate the association levels. Certainty of evidence was assessed using GRADE analysis. Statistical significance was set at $\mathrm{p}<0.05$.

\section{Results}

There were 37 potentially relevant publications,
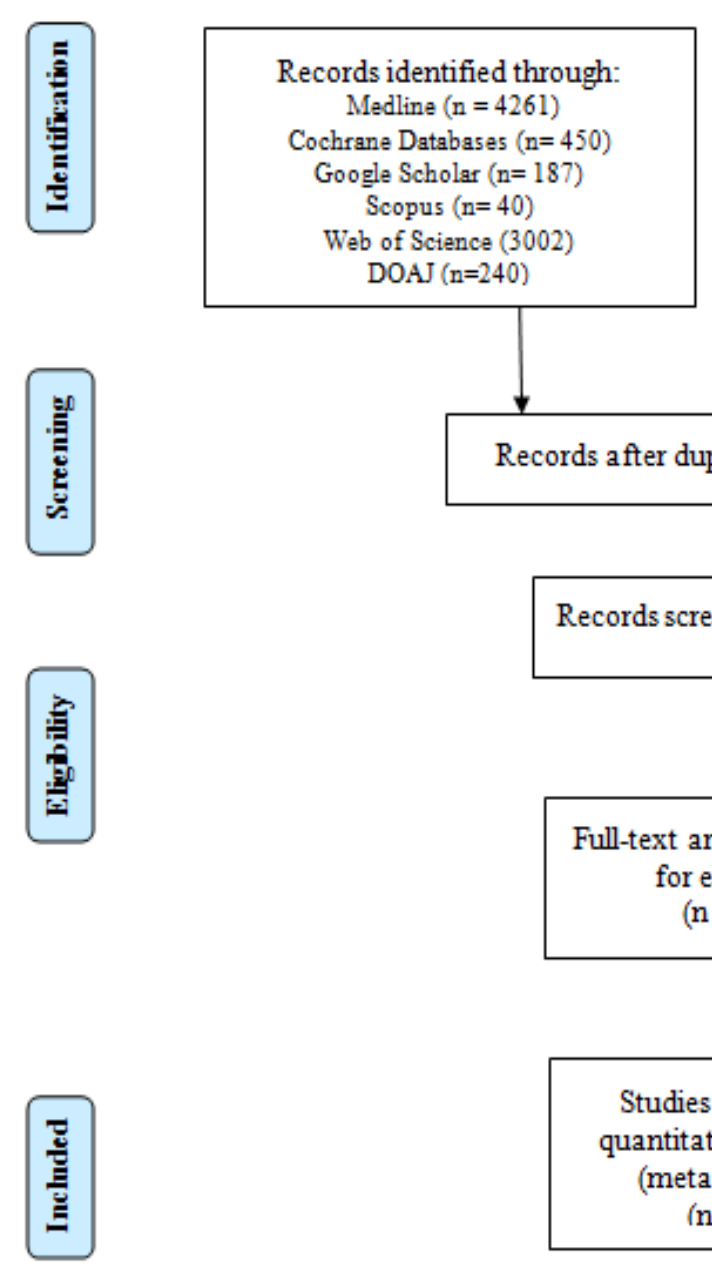

of which 14 were included in the review (Figure 1). Characteristics of the included studies are described in Table 1. There were 1352, 414 and 299 cases of oral cancer, OPMD, and OSMF respectively. The number of controls available for analysis with cases of oral cancer, OPMD, and OSMF were 11,699, 7,382 and 7,307 respectively. Table 2 presents the quality of studies that were included in the analysis. A low-to-moderate risk of bias was present for confounding variables. Additionally, a low risk of bias was present for incomplete outcome data and selective outcome reporting. Attrition levels were not recorded as there were no prospective studies.

Meta-analysis of studies that evaluated the asociation of type A blood group withoral cancer, OPMD, and OSMF are presented in Figure 2. Blood group A was significantly associated with both oral cancer(Odds ratio: 1.27[95\% CI, 1.10, 1.47], $\mathrm{P}=0.001$ ) and OPMD (Odds ratio: $1.33[95 \% \mathrm{CI}, 1.01,1.47], \mathrm{P}=0.04)$. However, no such association was noted between blood group A and OSMF. Sub-group analysis for the four OSMF studies reflected a significant reduction in $\mathrm{I}^{2}$ values from $76 \%$ to $51 \%(\mathrm{P}=0.11$; Figure 2$)$.

Meta-analysis of studies that evaluated the asociation of type $\mathrm{B}, \mathrm{AB}$, and $\mathrm{O}$ blood groups withoral cancer, OPMD, and OSMF are presented are presented in Figures 3, 4, and 5 respectively. No association was noted between blood group $\mathrm{B}$ and $\mathrm{AB}$ with oral cancer and OPMD. However, blood group $\mathrm{O}$ was significantly associated with lower

Additional records identified
through other sources $(n=2)$

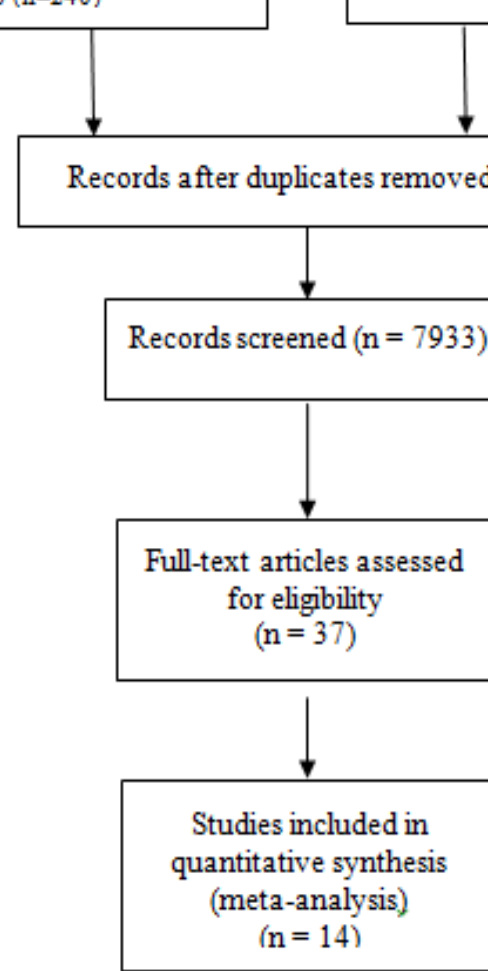

Figure 1. Flow Diagram of Study Selection Process 
Table 1. Characteristics of Studies Included in Meta-Analysis

\begin{tabular}{|c|c|c|c|c|c|}
\hline Study; Location & Study Design & $\begin{array}{c}\text { Number of } \\
\text { participants (n) }\end{array}$ & $\begin{array}{c}\text { ABO blood grouping among } \\
\text { Cases with Oral Cancer/ } \\
\text { OPMD/OSMF }\end{array}$ & $\begin{array}{l}\text { ABO blood grouping } \\
\text { among Controls with Oral } \\
\text { Cancer/OPMD/OSMF }\end{array}$ & Significance of findings \\
\hline \multicolumn{6}{|c|}{ Oral Cancer } \\
\hline $\begin{array}{l}\text { Gupta et al, } 2019 \\
\text { New Delhi, India }\end{array}$ & $\begin{array}{l}\text { Case control study } \\
\text { (Six months in 2019) }\end{array}$ & $\begin{array}{c}\text { Cases: } 76 \\
\text { Control: } 90\end{array}$ & $\begin{array}{l}\text { Type A - } 18(23.7 \%) \\
\text { Type B - } 23(30.1 \%) \\
\text { Type AB - } 25(32.9 \%) \\
\text { Type O - } 10(13.1 \%)\end{array}$ & $\begin{array}{l}\text { Type A - } 20(22.2 \%) \\
\text { Type B - } 33(36.7 \%) \\
\text { Type AB - } 11(12.2 \%) \\
\text { Type O - } 26(28.9 \%)\end{array}$ & $\begin{array}{l}\text { AB blood group associated } \\
\text { with significantly higher } \\
\text { chancers of oral cancer } \\
\text { O Blood group associated with } \\
\text { significantly lower chancer of } \\
\text { oral cancer }\end{array}$ \\
\hline $\begin{array}{l}\text { Hamed et al 2014, } \\
\text { Tehran, Iran }\end{array}$ & $\begin{array}{l}\text { Case control study } \\
\qquad(2013)\end{array}$ & $\begin{array}{l}\text { Cases: } 104 \\
\text { Control: } 90\end{array}$ & $\begin{array}{l}\text { Type A - } 25(24 \%) \\
\text { Type B - } 33(32 \%) \\
\text { Type AB -8 }(8 \%) \\
\text { Type O -38 }(36 \%)\end{array}$ & $\begin{array}{l}\text { Type A - } 24(27 \%) \\
\text { Type B - } 12(13 \%) \\
\text { Type AB - } 15(17 \%) \\
\text { Type O -39 }(43 \%)\end{array}$ & $\begin{array}{l}\text { B blood group associated with } \\
\text { significantly higher chancers of } \\
\text { oral cancer }\end{array}$ \\
\hline $\begin{array}{l}\text { Jaleel et al,2012 } \\
\text { Karnataka, India }\end{array}$ & $\begin{array}{l}\text { Case control study } \\
(2 \text { months in } 2010)\end{array}$ & $\begin{array}{l}\text { Cases: } 235 \\
\text { Control: } 812\end{array}$ & $\begin{array}{l}\text { Type A - } 68(29 \%) \\
\text { Type B - } 63(27 \%) \\
\text { Type AB - } 11(5 \%) \\
\text { Type O - } 93(39 \%)\end{array}$ & $\begin{array}{l}\text { Type A - } 177(23 \%) \\
\text { Type B - } 191(23 \%) \\
\text { Type AB - } 42(5 \%) \\
\text { Type O -402 }(50 \%)\end{array}$ & $\begin{array}{l}\text { A blood group associated with } \\
\text { significantly higher chancers of } \\
\text { oral cancer }\end{array}$ \\
\hline $\begin{array}{l}\text { Jalili et al, } 2018 \\
\text { Tabriz, Iran }\end{array}$ & $\begin{array}{l}\text { Case control study } \\
\text { (2011 to 2014) }\end{array}$ & $\begin{array}{c}\text { Cases: } 113 \\
\text { Control: } 2000\end{array}$ & $\begin{array}{l}\text { Type A - } 45(33.8 \%) \\
\text { Type B - } 24(18 \%) \\
\text { Type AB - } 26(19.5 \%) \\
\text { Type O - } 38(28.6 \%)\end{array}$ & $\begin{array}{l}\text { Type A - } 699(33.4 \%) \\
\text { Type B - } 191(23 \%) \\
\text { Type AB - } 468(23.4 \%) \\
\text { Type O - } 672(33.6 \%)\end{array}$ & $\begin{array}{l}\text { AB blood group had } \\
\text { significantly higher chances of } \\
\text { oral cancer } \\
\text { O blood group associated with } \\
\text { significantly lower chances of } \\
\text { oral cancer }\end{array}$ \\
\hline $\begin{array}{l}\text { Jamil et al, } 2006 \\
\text { Lahore, Pakistan }\end{array}$ & $\begin{array}{l}\text { Case control study } \\
\qquad(2006)\end{array}$ & $\begin{array}{l}\text { Cases: } 50 \\
\text { Control: } 50\end{array}$ & $\begin{array}{l}\text { Type A - } 16(32 \%) \\
\text { Type B - } 11(22 \%) \\
\text { Type AB - } 8(16 \%) \\
\text { Type O - } 15(30 \%)\end{array}$ & $\begin{array}{l}\text { Type A - } 14(28 \%) \\
\text { Type B - } 15(30 \%) \\
\text { Type AB - } 3(6 \%) \\
\text { Type O - } 18(36 \%)\end{array}$ & $\begin{array}{c}\text { No association noted between } \\
\text { ABO blood group and oral } \\
\text { cancer }\end{array}$ \\
\hline $\begin{array}{l}\text { Kumari et al, } 2017 \\
\text { Patna, India }\end{array}$ & $\begin{array}{l}\text { Case control study } \\
(2 \text { months in 2015) }\end{array}$ & $\begin{array}{l}\text { Cases: } 300 \\
\text { Control: } 800\end{array}$ & $\begin{array}{l}\text { Type A - } 92(30.7 \%) \\
\text { Type B - } 88(29.3 \%) \\
\text { Type AB - } 9(3 \%) \\
\text { Type O - } 111(37 \%)\end{array}$ & $\begin{array}{l}\text { Type A - } 181(22.6 \%) \\
\text { Type B - } 281(35.1 \%) \\
\text { Type AB - } 61(7.6 \%) \\
\text { Type O - } 277(34.6 \%)\end{array}$ & $\begin{array}{l}\text { A blood group significantly } \\
\text { associated with higher chances } \\
\text { of oral cancer }\end{array}$ \\
\hline $\begin{array}{l}\text { Oroei et al, } 2019 \\
\text { Iran }\end{array}$ & $\begin{array}{l}\text { Case control study } \\
\text { (2007 to 2017) }\end{array}$ & $\begin{array}{c}\text { Cases: } 190 \\
\text { Control: } 400\end{array}$ & $\begin{array}{l}\text { Type A - } 59(32.9 \%) \\
\text { Type B - } 38(22.4 \%) \\
\text { Type AB - } 13(7.6 \%) \\
\text { Type O - } 63(37.1 \%)\end{array}$ & $\begin{array}{l}\text { Type A - } 144(36 \%) \\
\text { Type B - } 74(18.5 \%) \\
\text { Type AB - } 33(8.2 \%) \\
\text { Type O - } 149(37.3 \%)\end{array}$ & $\begin{array}{l}\text { No association noted between } \\
\text { ABO blood group and oral } \\
\text { cancer }\end{array}$ \\
\hline $\begin{array}{l}\text { Poornima et al, } 2018 \\
\text { Telangana, India }\end{array}$ & $\begin{array}{l}\text { Retrospective Case- } \\
\text { Control study (2018) }\end{array}$ & $\begin{array}{l}\text { Cases: } 35 \\
\text { Control: } 30\end{array}$ & $\begin{array}{l}\text { Type A - } 6(17.1 \%) \\
\text { Type B - } 15(45.7 \%) \\
\text { Type AB - } 2(5 \%) \\
\text { Type O - } 11(31.4 \%)\end{array}$ & $\begin{array}{l}\text { Type A - } 9(30 \%) \\
\text { Type B - } 5(16.6 \%) \\
\text { Type AB - } 8(26.7 \%) \\
\text { Type O - } 8(26.7 \%)\end{array}$ & $\begin{array}{l}\text { B blood group associated with } \\
\text { significantly higher chances of } \\
\text { oral cancer }\end{array}$ \\
\hline $\begin{array}{l}\text { Shishodiaet al } 2019 \\
\text { Karnataka, India }\end{array}$ & $\begin{array}{l}\text { Case control study } \\
\quad(2013-2014)\end{array}$ & $\begin{array}{c}\text { Cases: } 35 \\
\text { Control: } 7027\end{array}$ & $\begin{array}{l}\text { Type A - } 13(37 \%) \\
\text { Type B - } 7(20 \%) \\
\text { Type AB - } 2(6 \%) \\
\text { Type O - } 13(37 \%)\end{array}$ & $\begin{array}{l}\text { Type A - } 1757(25 \%) \\
\text { Type B - } 2038(29 \%) \\
\text { Type AB - } 492(7 \%) \\
\text { Type O - } 2741(39 \%)\end{array}$ & $\begin{array}{l}\text { A blood group significantly } \\
\text { associated with higher chances } \\
\text { of oral cancer }\end{array}$ \\
\hline $\begin{array}{l}\text { Singh et al 2014, } \\
\text { New Delhi, India }\end{array}$ & $\begin{array}{l}\text { Case control study } \\
(6 \text { months in } 2013)\end{array}$ & $\begin{array}{l}\text { Cases: } 214 \\
\text { Control: } 7027\end{array}$ & $\begin{array}{l}\text { Type A - } 46(21.5 \%) \\
\text { Type B - } 70(32.7 \%) \\
\text { Type AB - } 19(8.87 \%) \\
\text { Type O - } 79(36.9 \%)\end{array}$ & $\begin{array}{l}\text { Type A - } 51(12.7 \%) \\
\text { Type B - } 142(35.5 \%) \\
\text { Type AB - } 47(11.7 \%) \\
\text { Type O - } 160(40 \%)\end{array}$ & $\begin{array}{l}\text { A blood group significantly } \\
\text { associated with higher chances } \\
\text { of oral cancer }\end{array}$ \\
\hline \multicolumn{6}{|c|}{ Oral Potentially Malignant Disorders (OPMD) } \\
\hline $\begin{array}{l}\text { Bhateja et al, } 2014 \\
\text { Pune, India }\end{array}$ & $\begin{array}{c}\text { Case- Control study } \\
\text { (2014) }\end{array}$ & $\begin{array}{l}\text { Cases: } 50 \\
\text { Control: } 50\end{array}$ & $\begin{array}{l}\text { Type A - } 36(18 \%) \\
\text { Type B - } 16(8 \%) \\
\text { Type AB - } 7(3.5 \%) \\
\text { Type O - } 16(8 \%)\end{array}$ & $\begin{array}{l}\text { Type A - } 13(26 \%) \\
\text { Type B - } 24(48 \%) \\
\text { Type AB - } 1(2 \%) \\
\text { Type O - } 12(24 \%)\end{array}$ & $\begin{array}{c}\text { A blood group significantly } \\
\text { associated with higher chances } \\
\text { of OPMD }\end{array}$ \\
\hline $\begin{array}{l}\text { Mehrotra et al, } 2017 \\
\text { Kanpur, India }\end{array}$ & $\begin{array}{l}\text { Cross-sectional study } \\
\text { with controls (2017) }\end{array}$ & $\begin{array}{l}\text { Cases: } 50 \\
\text { Control: } 50\end{array}$ & $\begin{array}{l}\text { Type A - } 11(21 \%) \\
\text { Type B }-26(52 \%) \\
\text { Type AB - } 6(13 \%) \\
\text { Type O - } 7(14 \%)\end{array}$ & $\begin{array}{l}\text { Type A - } 13(26 \%) \\
\text { Type B - } 18(35 \%) \\
\text { Type AB - } 9(19 \%) \\
\text { Type O - } 10(20 \%)\end{array}$ & $\begin{array}{l}\text { A and B blood group were } \\
\text { associated with significantly } \\
\text { higher chances of OPMD }\end{array}$ \\
\hline $\begin{array}{l}\text { Poornima et al, } 2018 \\
\text { Telangana, India }\end{array}$ & $\begin{array}{l}\text { Retrospective Case- } \\
\text { Control study (2018) }\end{array}$ & $\begin{array}{l}\text { Cases: } 35 \\
\text { Control: } 30\end{array}$ & $\begin{array}{l}\text { Type A - } 8(22.9 \%) \\
\text { Type B - } 14(40 \%) \\
\text { Type AB - } 5(14.9 \%) \\
\text { Type O - } 8(22.9 \%)\end{array}$ & $\begin{array}{l}\text { Type A - } 9(30 \%) \\
\text { Type B - } 5(16.6 \%) \\
\text { Type AB - } 8(26.7 \%) \\
\text { Type O - } 8(26.7 \%)\end{array}$ & $\begin{array}{l}\text { B blood group associated with } \\
\text { significantly higher chances of } \\
\text { OPMD }\end{array}$ \\
\hline $\begin{array}{l}\text { Rai et al, } 2015 \\
\text { Karnataka, India }\end{array}$ & $\begin{array}{l}\text { Cross-sectional study } \\
\text { with controls (2015) }\end{array}$ & $\begin{array}{l}\text { Cases: } 45 \\
\text { Control: } 45\end{array}$ & $\begin{array}{l}\text { Type A - } 19(42 \%) \\
\text { Type B - } 9(20 \%) \\
\text { Type AB - } 4(9 \%) \\
\text { Type O - } 13(29 \%)\end{array}$ & $\begin{array}{l}\text { Type A - } 17(38 \%) \\
\text { Type B - } 13(29 \%) \\
\text { Type AB - } 2(4 \%) \\
\text { Type O - } 13(29 \%)\end{array}$ & $\begin{array}{l}\text { No association noted between } \\
\text { ABO blood group and OPMD }\end{array}$ \\
\hline $\begin{array}{l}\text { Reddy et al, } 2016 \\
\text { Bhopal, India }\end{array}$ & $\begin{array}{l}\text { Cross-sectional study } \\
\text { with controls (2015) }\end{array}$ & $\begin{array}{l}\text { Cases: } 164 \\
\text { Control: } 180\end{array}$ & $\begin{array}{l}\text { Type A - } 33(20.1 \%) \\
\text { Type B - } 56(34.1 \%) \\
\text { Type AB - } 11(6.7 \%) \\
\text { Type O - } 64(39 \%)\end{array}$ & $\begin{array}{l}\text { Type A - } 42(23.3 \%) \\
\text { Type B - } 57(31.7 \%) \\
\text { Type AB - } 12(6.7 \%) \\
\text { Type O - } 69(38.3 \%)\end{array}$ & $\begin{array}{l}\text { No association noted between } \\
\text { ABO blood group and } \\
\text { OPMD. Participants with } \\
\text { blood group A were at higher } \\
\text { risk of developing OSMF in } \\
\text { comparison to others. }\end{array}$ \\
\hline $\begin{array}{l}\text { Shishodia et al, } \\
2019 \text { Karnataka, } \\
\text { India }\end{array}$ & $\begin{array}{l}\text { Case control study } \\
(6 \text { months in 2013) }\end{array}$ & $\begin{array}{c}\text { Cases: } 70 \\
\text { Control: } 7027\end{array}$ & $\begin{array}{l}\text { Type A - } 24(34.3 \%) \\
\text { Type B - } 20(28.6 \%) \\
\text { Type AB - } 4(2.8 \%) \\
\text { Type O - } 23(16.1 \%)\end{array}$ & $\begin{array}{l}\text { Type A - } 1757(25 \%) \\
\text { Type B - } 2038(29 \%) \\
\text { Type AB - } 492(7 \%) \\
\text { Type O - } 2741(39 \%)\end{array}$ & $\begin{array}{l}\text { No significant relationship } \\
\text { noted between ABO blood } \\
\text { group and OPMD }\end{array}$ \\
\hline
\end{tabular}


A.

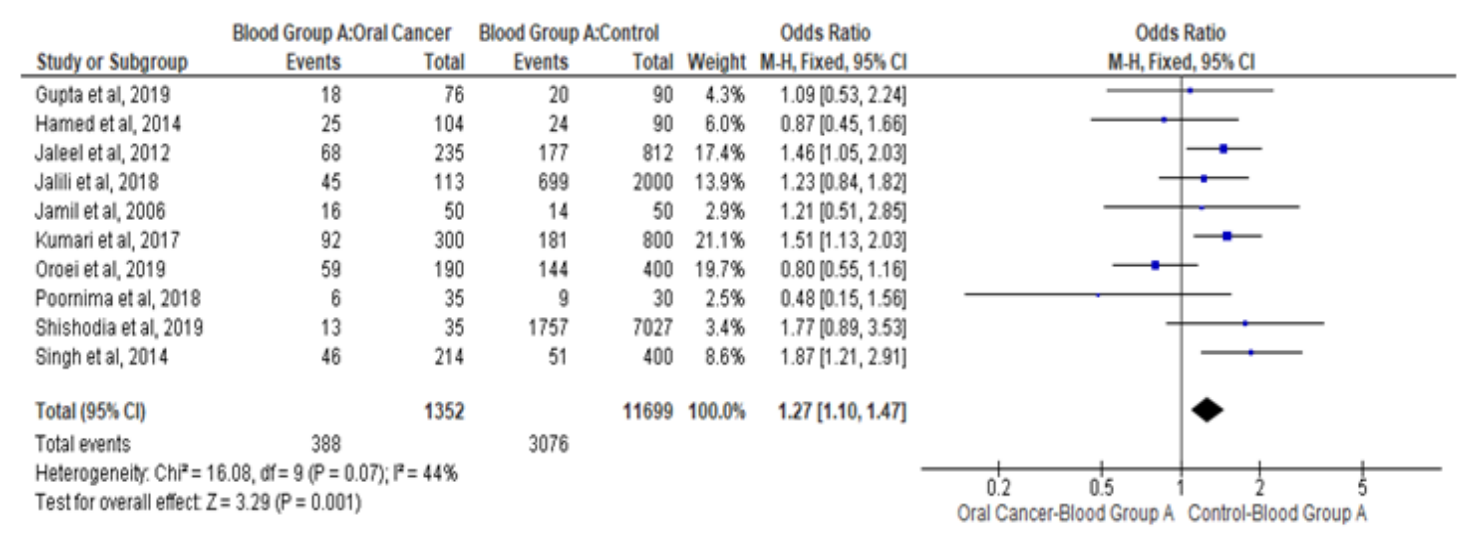

B.

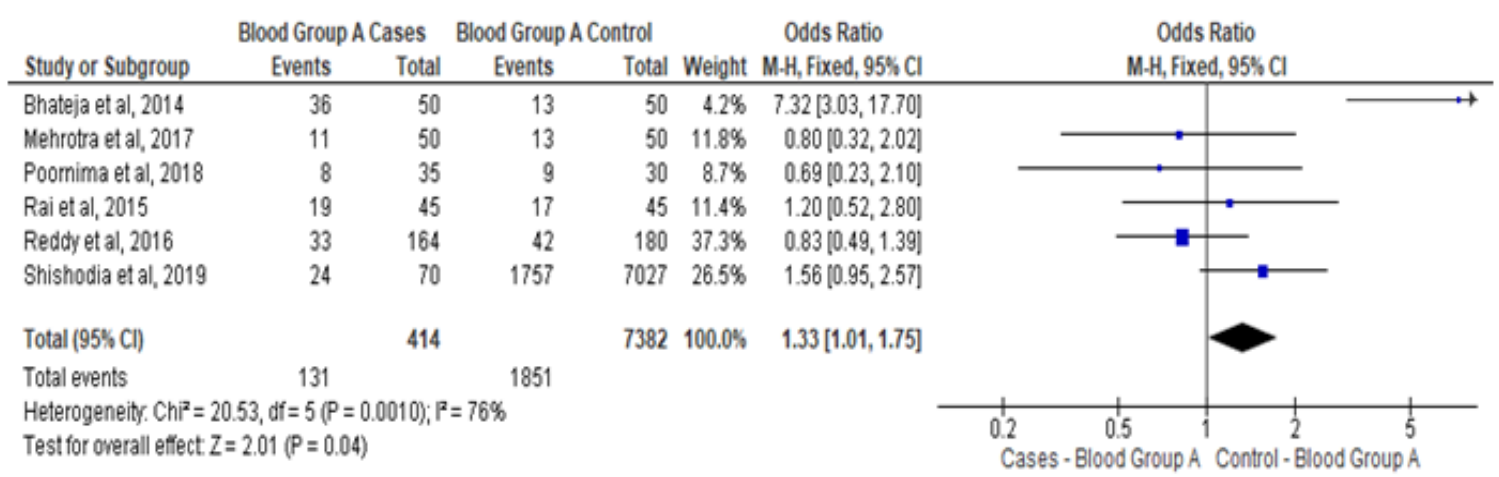

C.

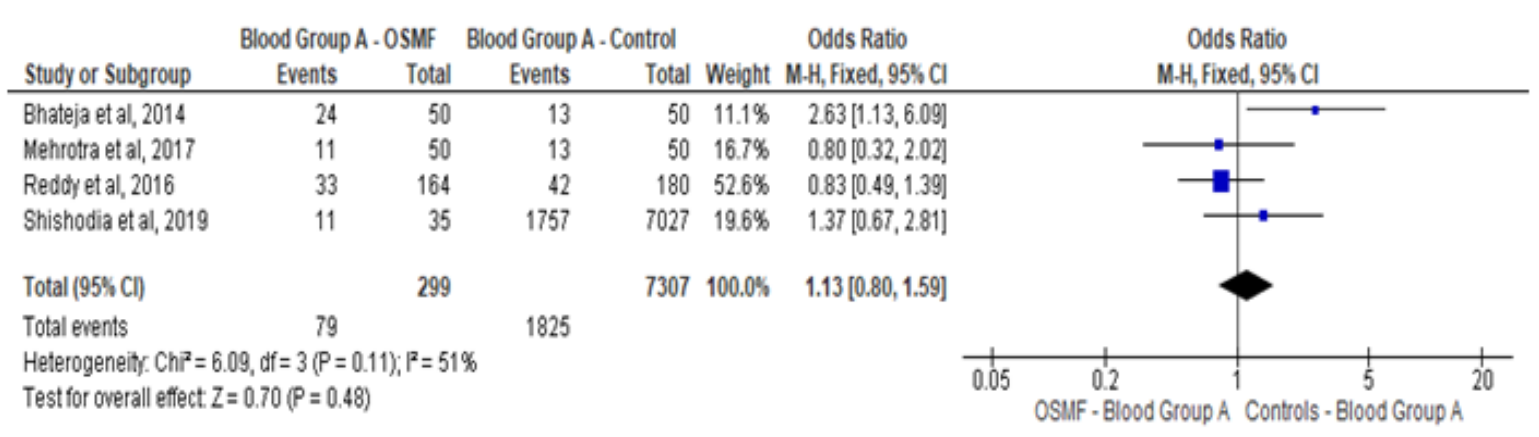

Figure 2. Type A Blood Group and Its Association between: Oral cancer patients with healthy controls (A) OPMD patients versus healthy controls (B), and OSMF patients versus healthy controls (C)

chances of oral cancer (Odds ratio: 0.81[95\% CI, 0.71, 0.93], $\mathrm{P}=0.002$; Figure 5). No such association was noted between blood group $\mathrm{O}$ with OPMD and OSMF.

The level of evidence obtained for the significant association of type $\mathrm{A}$ and type $\mathrm{O}$ blood group with oral cancer was moderate. Low level of evidence was achieved for the association between type A blood group and OPMD (Table 3).

Funnel plots were drawn for evaluating the studies included in the meta-analysis. The vertical line of the funnel plot represents the pooled mean effect size, and the dotted lines represent the $95 \%$ confidence interval (Figure 6).

\section{Discussion}

This meta-analysis is the first in scientific literature to assess the association between ABO blood groups and oral malignancy. Our meta-analysis included 14 trials and demonstrated a significant association between blood group A with oral cancer and OPMD. Blood group O was significantly associated with lower chances of oral cancer. No association was noted between blood group B and $\mathrm{AB}$ with oral cancer and OPMD. Also, no association could be found between ABO blood groups and OSMF.

Assessing the consistency of effects across studies is an essential part of a meta-analysis. The $\mathrm{I}^{2}$ statistic describes the percentage variation across studies that are 
Table 2. Quality Assessment of Studies Included in the Meta-Analysis

\begin{tabular}{|c|c|c|c|c|c|}
\hline Study Id & $\begin{array}{l}\text { Selection of } \\
\text { Participants }\end{array}$ & $\begin{array}{l}\text { Confounding } \\
\text { Variables }\end{array}$ & $\begin{array}{l}\text { Measurement } \\
\text { of Exposure }\end{array}$ & $\begin{array}{c}\text { Incomplete } \\
\text { Outcome Data }\end{array}$ & $\begin{array}{c}\text { Selective Outcome } \\
\text { Reporting }\end{array}$ \\
\hline Gupta et al, 2019 & $\mathrm{~L}$ & $\mathrm{M}$ & $\mathrm{L}$ & $\mathrm{L}$ & $\mathrm{L}$ \\
\hline Hamed et al, 2014 & $\mathrm{~L}$ & $\mathrm{~L}$ & $\mathrm{~L}$ & $\mathrm{~L}$ & $\mathrm{~L}$ \\
\hline Jaleel et al, 2012 & $\mathrm{~L}$ & M & $\mathrm{L}$ & $\mathrm{L}$ & $\mathrm{L}$ \\
\hline Jalili et al, 2018 & $\mathrm{~L}$ & M & $\mathrm{L}$ & $\mathrm{L}$ & $\mathrm{L}$ \\
\hline Jamil et al, 2006 & $\mathrm{~L}$ & M & $\mathrm{L}$ & $\mathrm{L}$ & $\mathrm{L}$ \\
\hline Kumari et al, 2017 & $\mathrm{~L}$ & M & $\mathrm{L}$ & $\mathrm{L}$ & $\mathrm{L}$ \\
\hline Oroei et al, 2019 & $\mathrm{~L}$ & $\mathrm{~L}$ & $\mathrm{~L}$ & $\mathrm{~L}$ & $\mathrm{~L}$ \\
\hline Poornima et al, 2018 & $\mathrm{~L}$ & M & $\mathrm{L}$ & $\mathrm{L}$ & $\mathrm{L}$ \\
\hline Shishodia et al, 2019 & $\mathrm{~L}$ & M & $\mathrm{L}$ & $\mathrm{L}$ & $\mathrm{L}$ \\
\hline Singh et al, 2014 & $\mathrm{~L}$ & M & $\mathrm{L}$ & $\mathrm{L}$ & $\mathrm{L}$ \\
\hline Bhateja et al, 2014 & $\mathrm{~L}$ & M & $\mathrm{L}$ & $\mathrm{L}$ & $\mathrm{L}$ \\
\hline Mehrotra et al, 2017 & $\mathrm{~L}$ & $\mathrm{~L}$ & $\mathrm{~L}$ & $\mathrm{~L}$ & $\mathrm{~L}$ \\
\hline Rai et al, 2015 & $\mathrm{~L}$ & $\mathrm{~L}$ & $\mathrm{~L}$ & $\mathrm{~L}$ & $\mathrm{~L}$ \\
\hline Reddy et al, 2016 & $\mathrm{~L}$ & M & $\mathrm{L}$ & $\mathrm{L}$ & $\mathrm{L}$ \\
\hline
\end{tabular}

L, Low risk of bias; M,: Moderate risk of bias; $\mathrm{H}$, High risk of bias; $\mathrm{U}$, Unclear risk of bias

caused by heterogeneity rather than chance. Heterogeneity is classified into low $(0-40 \%)$, moderate $(30-60 \%)$, substantial (50-90\%) and considerable (75-100\%) heterogeneity, as reported in Cochrane Handbook 5.1 [31]. $\mathrm{I}^{2}$ values of $44 \%$ and $76 \%$ were noted for association of blood group A with oral cancer and OPMD. However, in sub group analysis $\mathrm{I}^{2}$ value reduced to $51 \%$ for association between OSMF and blood group A. $\mathrm{I}^{2}$ values of $32 \%, 8 \%$ and $0 \%$ were noted for association between blood group $\mathrm{O}$ and oral cancer, OPMD, and OSMF.
The quality of evidence, as qualified by GRADE, was moderate for the association between oral cancer with blood group A and O. Level of evidence was low for association between blood group A with other potentially malignant disorders due to inconsistency in results $\left(\mathrm{I}^{2}\right.$ $=76 \%$ ). Level of evidence was also moderate to low for lack of association between blood groups $\mathrm{B}$ and $\mathrm{AB}$ with oral cancer and OPMD.

Oral malignancy is a serious health problem resulting in high morbidity and mortality. This study demonstrates

Table 3. GRADE Assessment for Certainty of Evidence for Association between Blood Groups with Oral Cancer and OPMD

\begin{tabular}{|c|c|c|c|c|c|c|c|c|c|c|}
\hline \multicolumn{6}{|c|}{ Certainty assessment } & \multicolumn{5}{|c|}{ No of patients } \\
\hline $\begin{array}{l}\text { No of } \\
\text { studies }\end{array}$ & Study design & $\begin{array}{l}\text { Risk of } \\
\text { bias }\end{array}$ & Inconsistency & Indirectness & Imprecision & $\begin{array}{c}\text { Other } \\
\text { considerations }\end{array}$ & $\begin{array}{l}\text { Malnourished } \\
\text { children }\end{array}$ & $\begin{array}{c}\text { Well } \\
\text { Nourished } \\
\text { children }\end{array}$ & $\begin{array}{l}\text { Relative } \\
(95 \% \mathrm{CI})\end{array}$ & Certainty \\
\hline \multicolumn{11}{|c|}{ Type A blood group and its association with oral cancer } \\
\hline 10 & $\begin{array}{l}\text { Observational } \\
\text { studies }\end{array}$ & Serious & Not serious & Not serious & Not serious & $\begin{array}{c}\text { Strong } \\
\text { association, } \\
\text { all plausible } \\
\text { residual } \\
\text { confounding } \\
\text { would suggest } \\
\text { spurious effect }\end{array}$ & $\begin{array}{c}388 / 1352 \\
(28.6 \%)\end{array}$ & $\begin{array}{c}3076 / 11699 \\
(26.3 \%)\end{array}$ & $\begin{array}{c}\text { OR } 1.27 \\
(1.10 \text { to } \\
1.47)\end{array}$ & $\begin{array}{l}\bigoplus \bigoplus \bigoplus \\
\text { MODERATE }\end{array}$ \\
\hline \multicolumn{11}{|c|}{ Type 0 blood group and its association with oral cancer } \\
\hline 10 & $\begin{array}{l}\text { Observational } \\
\text { studies }\end{array}$ & Serious & Not serious & Not serious & Not serious & $\begin{array}{l}\text { Strong } \\
\text { association, } \\
\text { all plausible } \\
\text { residual } \\
\text { confounding } \\
\text { would } \\
\text { reduce the } \\
\text { demonstrated } \\
\text { effect }\end{array}$ & $\begin{array}{c}459 / 1352 \\
(33.9 \%)\end{array}$ & $\begin{array}{c}4492 / 11699 \\
(38.3 \%)\end{array}$ & $\begin{array}{c}\text { OR } 0.81 \\
(0.71 \text { to } \\
0.93)\end{array}$ & $\begin{array}{l}\bigoplus \bigoplus \bigoplus \\
\text { MODERATE }\end{array}$ \\
\hline \multicolumn{11}{|c|}{ Type A blood group and its association with oral potentially malignant disorders (OPMD) } \\
\hline 6 & $\begin{array}{l}\text { Observational } \\
\text { studies }\end{array}$ & Serious & Serious & Not serious & Not serious & $\begin{array}{c}\text { Strong } \\
\text { association, } \\
\text { all plausible } \\
\text { residual } \\
\text { confounding } \\
\text { would suggest } \\
\text { spurious effect }\end{array}$ & $\begin{array}{l}131 / 414 \\
(31.6 \%)\end{array}$ & $\begin{array}{c}1851 / 7382 \\
(25 \%)\end{array}$ & $\begin{array}{c}\text { OR } 1.33 \\
(1.01 \text { to } \\
1.75)\end{array}$ & $\begin{array}{l}\oplus \bigoplus \\
\text { LOW }\end{array}$ \\
\hline
\end{tabular}


A.

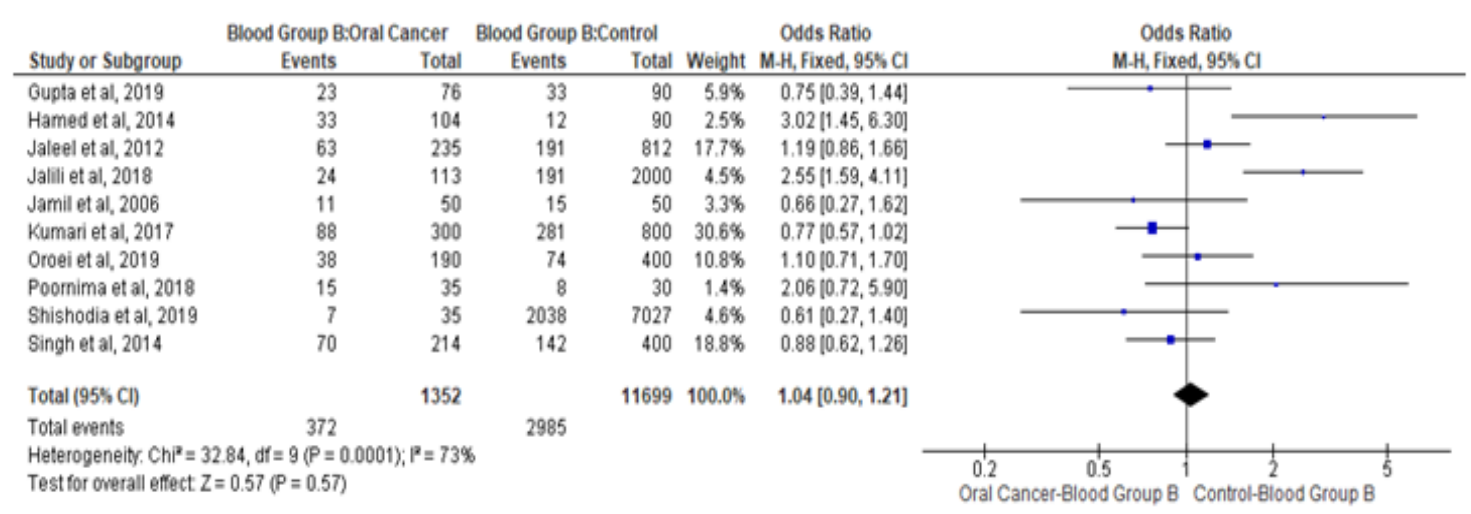

B.

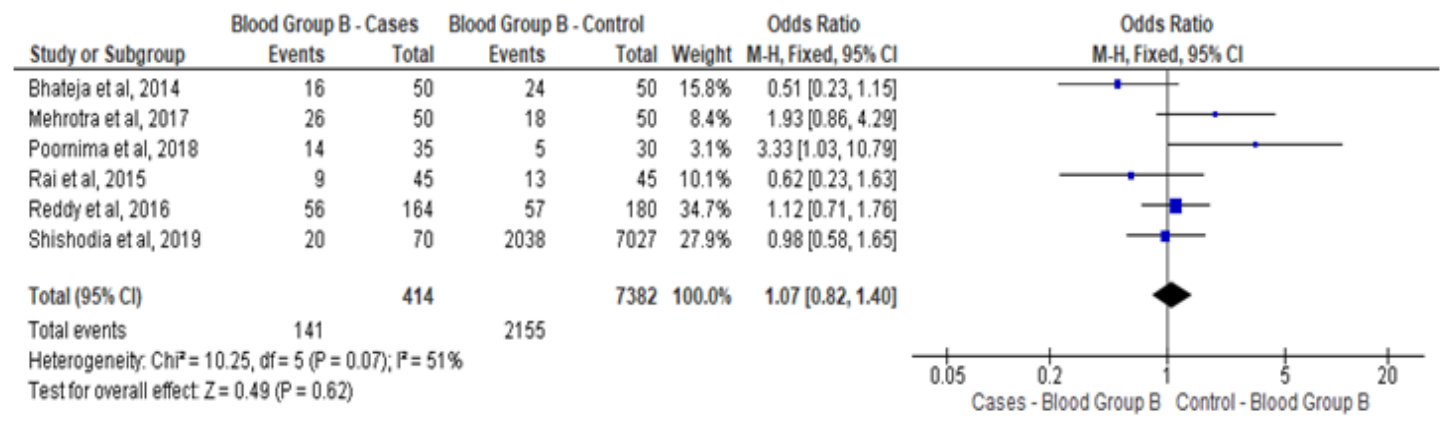

C.

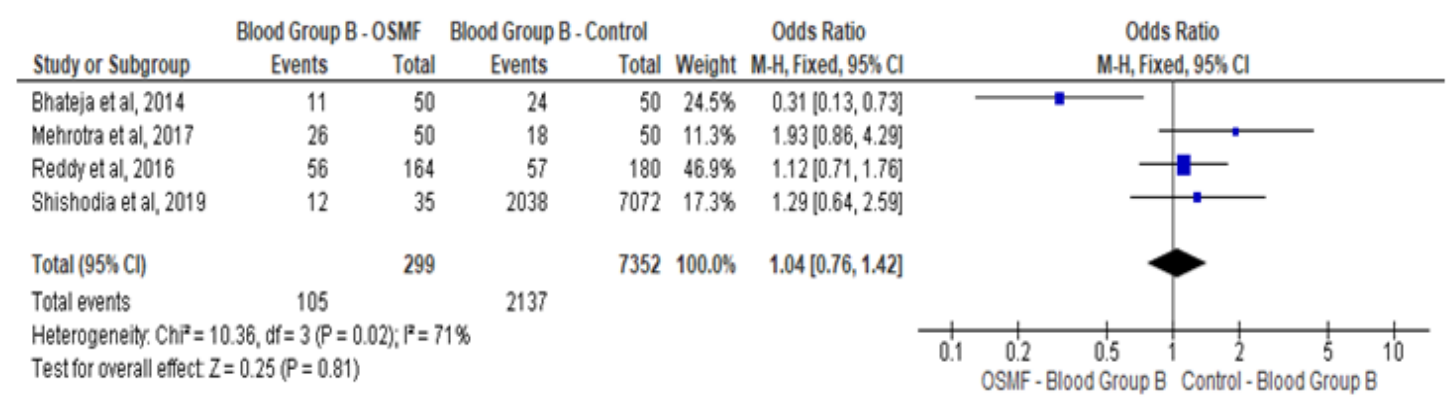

Figure 3. Type B Blood Group and Its Association between: Oral cancer patients with healthy controls (A) OPMD patients versus healthy controls (B), and OSMF patients versus healthy controls (C)

that there exists a relationship between $\mathrm{ABO}$ blood groups and oral cancer; specifically, blood group A and $\mathrm{O}$. Health care providers in cancer screening and preventive programs should take these findings into considerations. Blood typing also can be used as a method to identify the susceptible individuals and counsel them.

World Health Organization predicts that global cancer deaths are projected to increase by $45 \%$ between 2008 and 2030 (WHO, 2020). Population projections by census bureau predict a $35 \%$ oral cancer incidence increase in United States by 2030 from the 2010 level (Smith et al., 2009). Oral cancer is largely curable if detected early and treated appropriately. Knowledge about the factors and conditions associated with oral cancer allows the construction of models to understand their development and to collaborate in the creation of evidence based public policies.

We analysed, descriptive studies with controls, case control studies and retrospective studies with control together. However, although it is good practice to analyse data by study design, analysing for study design separately would have reduced the number of studies in each analysis to a number that impedes any meaningful conclusion. Several studies included in the analysis involved controls from large groups of blood donors. Even through these volunteer donors are considered to be representative of their study's ethnic compositions, they may have other characteristics associated with altered risk of oral cancer. Limitations of the present study also include a limited number of analysed studies, and the study protocol was not registered. The present study was intended to fill the knowledge gap and to provide information and evidence 
A.

\begin{tabular}{|c|c|c|c|c|c|c|c|c|c|c|c|}
\hline \multirow[b]{2}{*}{ Study or Subgroup } & \multicolumn{2}{|c|}{ BloodGroup AB:Oral Cancer } & \multicolumn{2}{|c|}{ BloodGroup AB:Control } & & \multirow{2}{*}{$\begin{array}{c}\text { Odds Ratio } \\
\text { M-H, Fixed, } 95 \% \mathrm{Cl}\end{array}$} & \multirow{2}{*}{\multicolumn{5}{|c|}{$\begin{array}{c}\text { Odds Ratio } \\
\text { M-H, Fixed, } 95 \% \text { Cl }\end{array}$}} \\
\hline & Events & Total & Events & Total & Weight & & & & & & \\
\hline Gupta et al, 2019 & 25 & 76 & 11 & 90 & $4.0 \%$ & $3.52[1.60,7.77]$ & & & & - & \\
\hline Hamed et al, 2014 & 8 & 104 & 15 & 90 & $8.7 \%$ & $0.42[0.17,1.03]$ & & & & & \\
\hline Jaleel et al, 2012 & 11 & 235 & 42 & 812 & $10.6 \%$ & $0.90[0.46,1.78]$ & & & & & \\
\hline Jalili et al, 2018 & 38 & 113 & 468 & 2000 & $19.5 \%$ & $1.66[1.11,2.48]$ & & & & $\longrightarrow$ & \\
\hline Jamil et al, 2006 & 8 & 50 & 3 & 50 & $1.5 \%$ & $2.98[0.74,11.99]$ & & & & & \\
\hline Kumari et al, 2017 & 9 & 300 & 61 & 800 & $19.0 \%$ & $0.37[0.18,0.76]$ & & & & & \\
\hline Oroei et al, 2019 & 13 & 190 & 33 & 400 & $11.6 \%$ & $0.82[0.42,1.59]$ & & & & & \\
\hline Poomima et al, 2018 & 2 & 35 & 8 & 30 & $4.8 \%$ & $0.17[0.03,0.86]$ & & & & & \\
\hline Shishodia et al, 2019 & 2 & 35 & 492 & 7027 & $2.7 \%$ & $0.81[0.19,3.36]$ & & & & & \\
\hline Singh et al, 2014 & 19 & 214 & 47 & 400 & $17.6 \%$ & $0.73[0.42,1.28]$ & & & & & \\
\hline Total $(95 \% \mathrm{Cl})$ & & 1352 & & 11699 & $100.0 \%$ & $0.96[0.78,1.20]$ & & & & & \\
\hline Total events & 135 & & 1180 & & & & & & & & \\
\hline $\begin{array}{l}\text { Heterogeneity: } \mathrm{Ch}^{\mathbf{2}}=3 \\
\text { Test for overall effect } \mathrm{Z}\end{array}$ & $\begin{array}{l}.43, d f=9(P<0 \\
=0.33(P=0.74)\end{array}$ & $=75 \%$ & & & & & $\begin{array}{c}0.05 \\
\text { Oral }\end{array}$ & 0.2 & oup $A B$ & $\begin{array}{c}5 \\
\text { Control-8loodGroup AB }\end{array}$ & 20 \\
\hline
\end{tabular}

B.

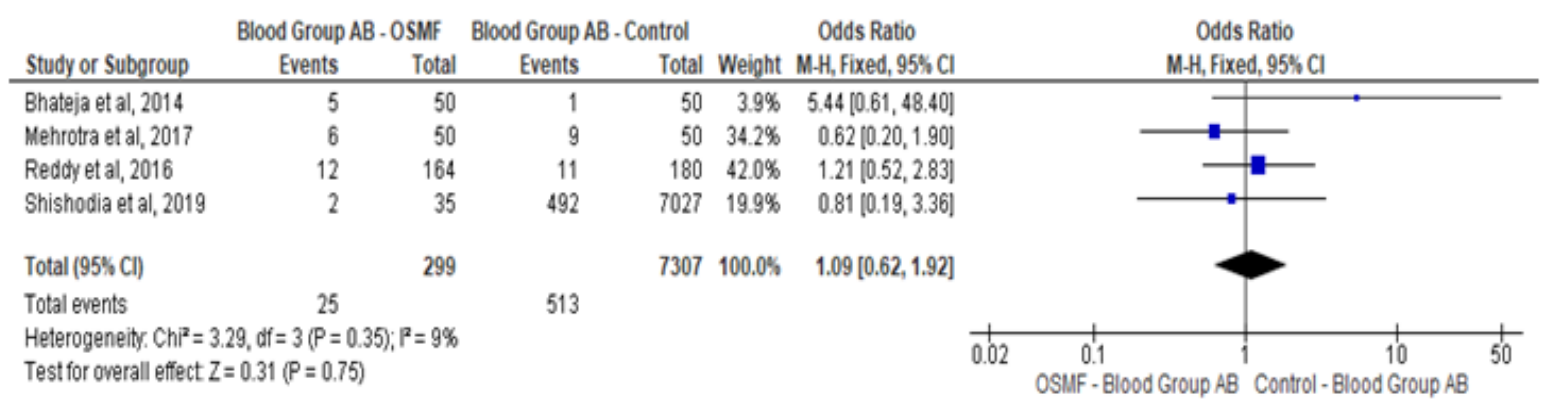

C.

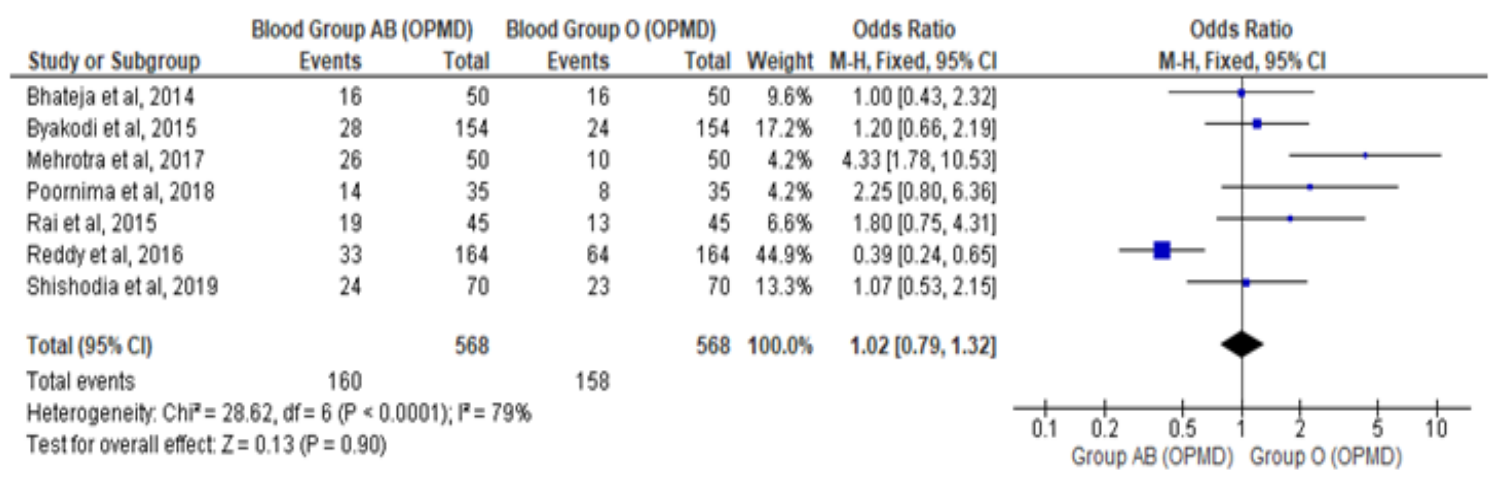

Figure 4. Type AB Blood Group and Its Association between: Oral cancer patients with healthy controls (A) OPMD patients versus healthy controls (B), and OSMF patients versus healthy controls (C)

for the association between $\mathrm{ABO}$ blood group system with oral cancer and other potentially malignant disorders. However, further prospective research on the association between blood groups with oral malignancy and potential malignant disorders is required.

In conclusion, this study demonstrates that there exists a relationship between $\mathrm{ABO}$ blood groups and oral cancer. Meta-analysis suggests A blood group has a greater risk for developing oral cancer and OPMD. Blood group $\mathrm{O}$ was associated with lower chances of oral cancer. No association was noted between $\mathrm{ABO}$ blood group system with OSMF.

\section{Author Contribution Statement}

AS and BMP contributed equally to study design, study selection, data extraction, statistical analysis, draft preparation and approval of the final version.

\section{Acknowledgments}

Compliance with Ethical Standards. Ethical approval: Not Applicable - Review Article. This article does not contain any studies with human participants performed by any of the authors. 
A.

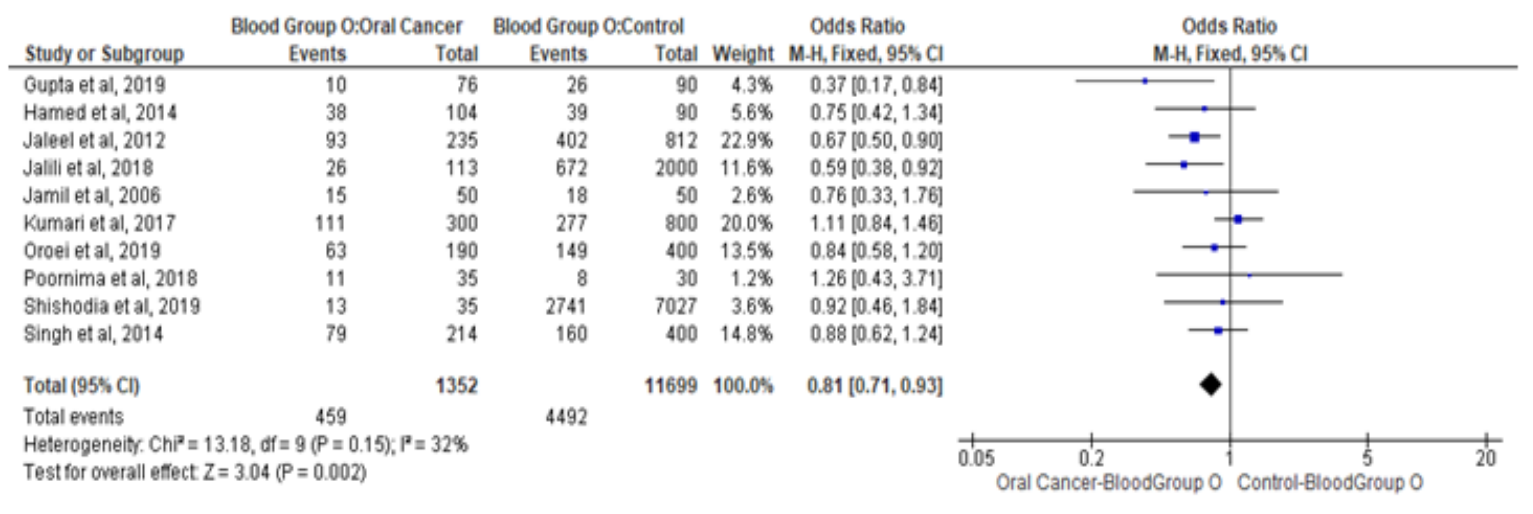

B.

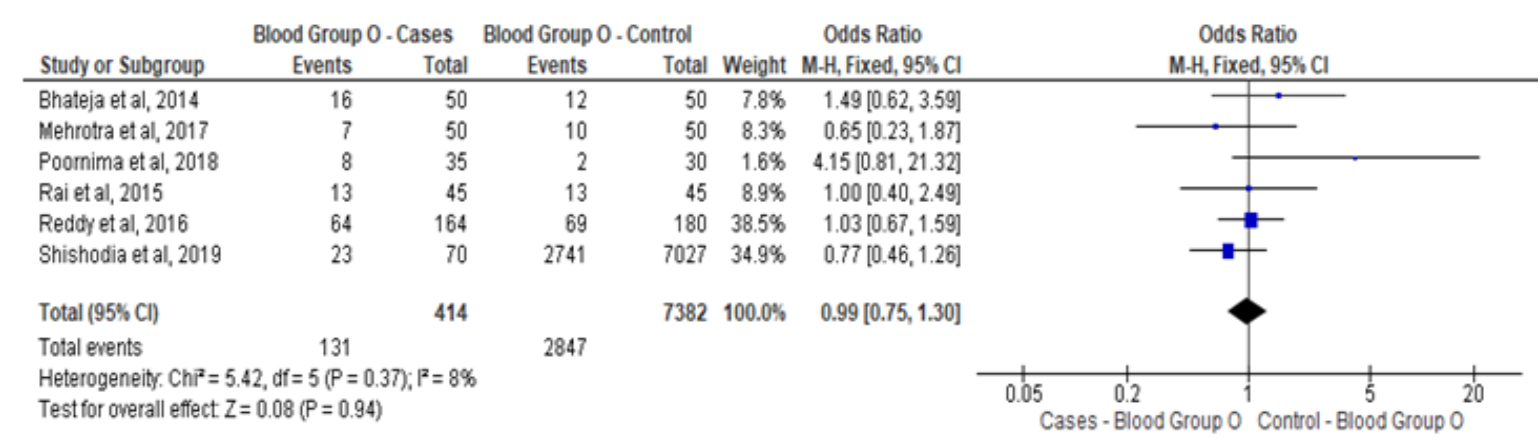

C.

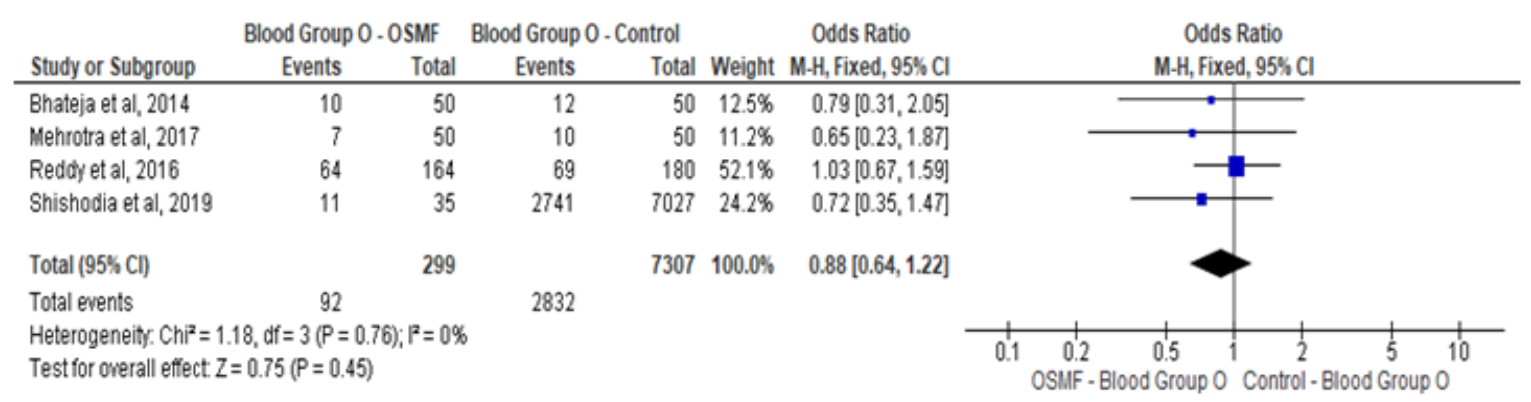

Figure 5. Type O Blood Group and Its Association between: Oral cancer patients with healthy controls (A) OPMD patients versus healthy controls (B), and OSMF patients versus healthy controls (C)

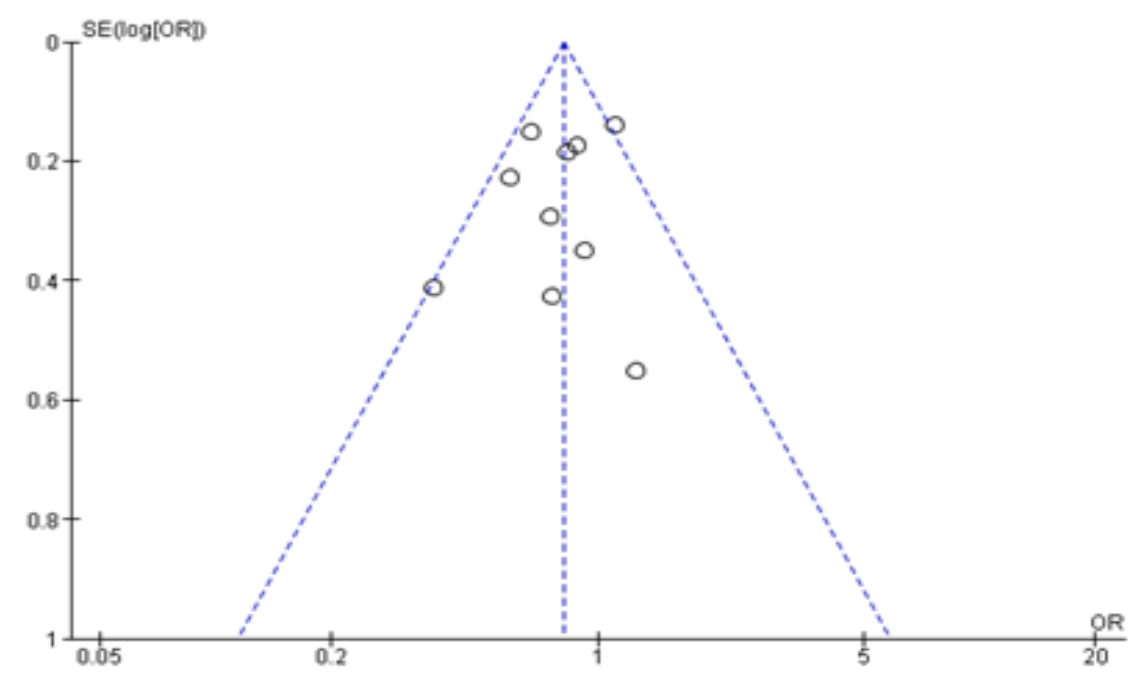

Figure 6. Funnel Plot of Included Studies Evaluating ABO Blood Group and Its Association with Oral Cancer 
Conflict of Interest

Authors declare that there is no conflict of interests

\section{References}

Anderson E, Haas C (1984). Blood type A and familial breast cancer. Cancer, 54, 1845-9.

Beckman L, Angqvist A (1987). On the mechanism behind the association between $\mathrm{ABO}$ blood groups and gastric carcinoma. Hum Hered, 37, 140-3.

Bhateja S, Arora G (2014). ABO blood groups and oral premalignancies: A clinical study in selected Indian population. Indian J Cancer, 51, 219-21.

Celic D, Lipozencic J, Kolaric B, et al (2019). Association between blood group and nonmelanoma skin cancers (Basal Cell Carcinoma and Squamous Cell Carcinoma). Int $J$ Environ Res Public Health, 16, 2267.

Dabelsteen E, Gao S (2005). ABO blood group antigens in oral cancer. $J$ Dent Res, 8, 21-8.

Dadhich M, Prabhu V, Pai R, et al (2014). Serum and salivary sialic acid as a biomarker in oral potentially malignant disorders and oral cancer. Indian J Cancer, 51, 2148.

Gupta D, Chaudhary N, Makkar S, Gupta A(2020). Relationship of ABO blood group and oral cancer: a study in selected group of Indian patients. Available from: "www.npplweb. com > wjsr > content > wjsr_8_1" Accessed July 10, 2020.

Huang T, Poole M, Okereke I, et al (2015). Depression and risk of epithelial ovarian cancer: Results from two large prospective cohort studies. Gynecol Oncol, 139, 481-6.

Henderson J, Seagrott V, Golgacre M (1993) Ovarian cancer and ABO blood groups. J Epidemiol Common Health, 47, 287-9.

Indian council of medical research and National centre for disease informatics and research (2020). Report of National Cancer Registry Programme. Available from: "www. ncdirindia.org" Accessed July 10, 2020.

Jaleel F, Nagarajappa R (2012). Relationship between ABO blood groups and oral cancer. Indian J Dent Res, 23, 7-12.

Jalili L, Zarabadipour M, Azmoudeh F, Esfehani M, Tamiz P (2018). ABO blood group distribution in patients with oral squamous cell carcinoma. Ann Dent Specialty, 6, 149-3.

Jamil S, Saleem N, Amjad S, et al (2006). Relationship of ABO blood groups in patients with oral cancer. Annals, 12, 104-5.

Kim Y, Park E, Lee J, et al (2013). Testing a tool for assessing the risk of bias for nonrandomized studies showed moderate reliability and promising validity. $J$ Clin Epidemiol, 66, 408-4.

Kumari P, Ramesh V, Islam F (2017). Association between ABO blood group and oral cancer - a retrospective hospital-based study in Patna, Bihar. Int J Recent Sci Res, 8, 19186.

Mao Y, Yang W, Qi Q, et al (2019). Blood groups A and AB are associated with increased gastric cancer risk: evidence from a large genetic study and systematic review. $B M C$ Cancer, 19, 164.

Mehrotra V, Garg K, Singh K, Singh R, Singh V (2017) A cross sectional study based on assessment of association between $\mathrm{ABO}$ blood group and oral submucous fibrosis. Rama Univ $J$ Dent Sci, 4, 1-5.

Miao S, Zhou W, Chen L, Wang S, Liu X (2014). Influence of $\mathrm{ABO}$ blood group and Rhesus factor on breast cancer risk: A meta-analysis of 9665 breast cancer patients and 244,768 controls. Asia Pac J Clin Oncol, 10, 101-8.

Mortazavi H, Hajian S, Fadavi E, et al (2014). ABO blood groups in oral cancer: a first case- control study in a defined group of Iranian patients. Asian Pac J Cancer Prev, 15, 1415-8.

Oroei M (2019). Determining the association of benign and malignant salivary gland tumours with ABO-Rh blood groups. Bengal J Otolaryngol Head Neck Surg, 27, 154.
Poornima R, Shyam N, Kiran G, et al (2018). Evaluation of ABO blood groups in oral potentially malignant disorders and oral squamous cell carcinoma - a retrospective study in south Indian population. Indian J Mednodent Allied Sci, 6, 66.

Rai P, Acharya S, Hallikeri K (2015). Assessment of ABO blood grouping and secretor status in The saliva of the patients with oral potentially malignant disorders. J Oral Maxillofac Pathol, 19, 164-9.

Reddy V, Moon J, Sharva V, et al (2016.) Is there an association between oral submucous fibrosis and $\mathrm{ABO}$ blood grouping?. $J$ Cancer Res Ther, 12, 126.

Sankaranarayanan R, Ramadas K, Amarasinghe H, et al (2015). Oral Cancer: prevention, early detection, and treatment, chapter 5. In: Cancer: Disease Control Priorities, Third Edition. Washington (DC): The International Bank for Reconstruction and Development / The World Bank, 2015.

Schünemann H, Brozek J, Guyatt G, Oxman A (2013). The GRADE handbook, 2013. Available at: "https://training. cochrane.org/resource/grade-handbook". Accessed July 5, 2020.

Shah M, Telang S, Raval G, Shah P, Patel S (2008). Serum fucosylation changes in oral cancer and oral precancerous conditions a-L- Fucosidase as a marker. Cancer, 113, 336-6.

Shishodia N, Anekar J, Raj A, et al (2018). Insight on the relationship of $\mathrm{ABO}$ blood grouping associated with oral premalignant lesions, conditions and inherited oral cancer syndromes. J Exp Ther Oncol, 2018, 1-5.

Singh K, Kote S, Patthi B, et al (2014). Relative risk of various head and neck cancers among different blood groups: an analytical study. J Clin Diagn Res, 8, 25-8.

Smith B, Smith G, Hurria A, Hortobagyi G, Buchholz T (2009). Future of Cancer Incidence in the United States: Burdens Upon an Aging, Changing Nation. J Clin Oncol, 27, 2758-5.

The Cochrane Collaboration (2011).Cochrane handbook for systematic reviews of interventions, 2011. Available form: "http://handbook.cochrane.org". Accessed July 1, 2020.

Vasan K, Hwang J, Rostgaard K, et al (2016). ABO blood group and risk of cancer: A register- based cohort study of 1.6 million blood donors. Cancer Epidemiol, 44, 40-3.

Wanakulasuriya S, Johnson W, van der Waal I (2007). Nomenclature and classification of potentially malignant disorders of the oral mucosa. J Oral Pathol Med, 36, 575.

Warnakulasuriya S (2009). Global epidemiology of oral and oropharyngeal cancer. Oral Oncol, 45, 309-6.

World Health Organization. Oral cancer (2020). Available from: "https://www.who.int/cancer/prevention/diagnosisscreening/oral-cancer/en/" Accessed July 10, 2020.

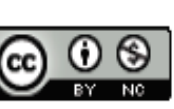

This work is licensed under a Creative Commons AttributionNon Commercial 4.0 International License. 